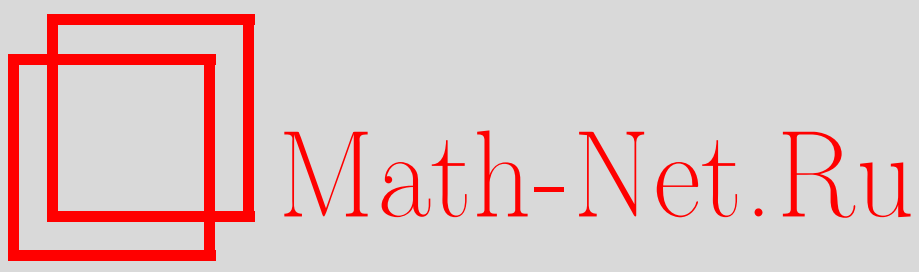

А. Ф. Заусаев, А. А. Заусаев, А. Г. Ольхин, Численное интегрирование уравнений движения больших планет (Меркурий-Плутон) и Луны с учетом радиолокационных наблюдений, Вестн. Сам. гос. техн. ун-та. Сер. Физ.-мат. науки, 2004, выпуск 26, 43-47

DOI: https://doi.org/10.14498/vsgtu175

Использование Общероссийского математического портала Math-Net.Ru подразумевает, что вы прочитали и согласны с пользовательским соглашением http://www.mathnet.ru/rus/agreement

Параметры загрузки:

IP : 3.82 .47 .9

26 апреля 2023 г., 17:38:12

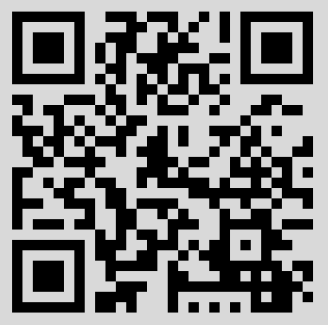




\section{ЧИСЛЕННОЕ ИНТЕГРИРОВАНИЕ УРАВНЕНИЙ ДВИЖЕНИЯ БОЛЬШИХ ПЛАНЕТ (МЕРКУРИЙ-ПЛУТОН) И ЛУНЫ С УЧЕТОМ РАДИОЛОКАЦИОННЫХ НАБЛЮДЕНИЙ}

Проведено численное интегрирование уравнений движения больших планет (Меркурий-Плутон) $и$ Луны на интервале времени с 1950 по 2000 г2. В уравнениях движения Луны от несферичности Земли учитывались лишь зональные гармоники. Результаты вычислений для внутренних планет (Меркурий-Марс) сопоставлены с радиолокационными наблюдениями. Получено вполне удовлетворительное согласие численной теории с результатами наблюдений.

В настоящее время имеется ряд высокоточных численных теорий движения больших планет, согласующихся с радиолокационными наблюдениями [1-4]. Наиболее точной из них является теория планетных и Лунных эфемерид DE405. Однако точные положения планет охватывают лишь интервал времени с 1600 по 2200 гг. В численной теории движения планет и Луны DE406 координаты планет вычислены на интервале времени с 3000 г. до н.э. по 3000 г. н.э. Недостатком, на наш взгляд, данной теории является большой объем внешней памяти (около 200 мегабайт), в которых хранится информация об эфемеридах планет в форме полиномов Чебышева на интервале времени 6000 лет. Целью наших исследований является получение в дальнейшем банка данных координат больших планет на интервале времени порядка 20 и более тысяч лет и применение этого банка данных к исследованию эволюции орбит комет и астероидов.

Точность вычисления элементов орбит комет и астероидов значительно ниже, чем элементов орбит больших планет, поэтому нет необходимости в получении координат планет с той же степенью точности, как в DE405 или DE406. Тем не менее, ошибки в координатах и скоростях больших планет на исследуемом интервале времени не должны быть значительными. Вследствие того, что при исследовании эволюции орбит малых тел Солнечной системы учитываются, в основном, гравитационные взаимодействия, а также релятивистские эффекты, следует оценить величину ошибок, которые могут возникнуть в процессе интегрирования больших планет путем сопоставления координат с радиолокационными наблюдениями.

Радиолокационные наблюдения планет, проведенные как в СССР, так и в США [5-7], позволили значительно уточнить величину астрономической единицы, а также построить численные теории движения внутренних планет и Луны, на два-три порядка превосходящие по точности теории, основанные на оптических наблюдениях.

Радиолокационный метод позволяет измерить топоцентрическое расстояние центра видимого диска планеты и радиальную скорость изменения этого расстояния.

Полагаем, что электромагнитное излучение покинуло передающую антенну в момент $t_{1}$, достигло центра видимого диска планеты в момент $t_{2}$ и, отразившись, вернулось на Землю к приемной антенне в момент $t_{3}$ (рис. 1). Обозначая векторами $r_{1}\left(t_{1}\right), r_{2}\left(t_{2}\right)$ и $r_{3}\left(t_{3}\right)$ положение передающей антенны, центра масс планеты и приемной антенны, центра масс планеты в соответствующие моменты времени в гелиоцентрической системе координат, получим полное время

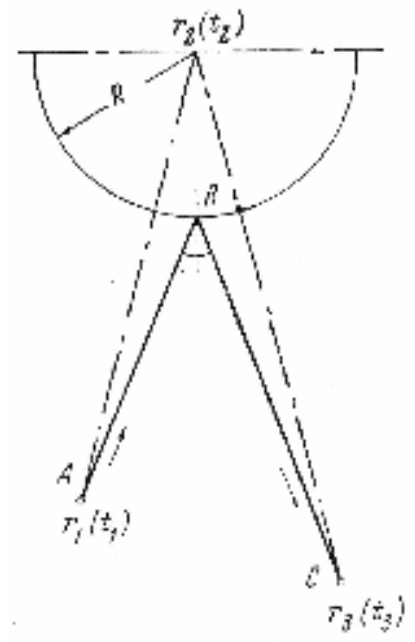

$\mathrm{P}$ и с. 1. Путь падающего $(A B)$ и отраженного $(B C)$ лучей при радиолокации. Вследствие малости угла АВС принято

$$
\begin{aligned}
& A B \cong\left|r_{2}\left(t_{2}\right)-r_{1}\left(t_{1}\right)\right|-R, \\
& B C \cong\left|r_{3}\left(t_{3}\right)-r_{2}\left(t_{2}\right)\right|-R .
\end{aligned}
$$
распространения волн $\left(\Delta t=t_{3}-t_{1}\right)$ по формуле [7]:

$$
\Delta t=\frac{1}{c}\left[\left|r_{2}\left(t_{2}\right)-r_{1}\left(t_{1}\right)\right|+\left|r_{3}\left(t_{3}\right)-r_{2}\left(t_{2}\right)\right|-2 R\right],
$$

где $c$-скорость света в вакууме, R-радиус поверхности планеты.

Полагая

$$
\underset{12}{\alpha}=\frac{d}{d t}\left|r_{2}\left(t_{2}\right)-r_{1}\left(t_{1}\right)\right|, \underset{23}{\&}=\frac{d}{d t}\left|r_{3}\left(t_{3}\right)-r_{2}\left(t_{2}\right)\right|,
$$


определим полное доплеровское смещение частоты сигнала как

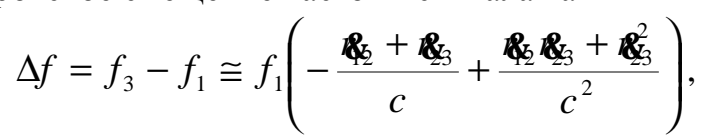

где $f_{1}$-частота начального излучения.

На основе радиолокационных наблюдений как в нашей стране, так и в США сотрудники ИТА РАН г. Санкт-Петербурга составили банк данных радионаблюдений Меркурия, Венеры и Mapca [4]. Основные сведения, содержащие результаты измерения времени излучения, времени запаздывания, доплеровского смещения; координаты передающей и приемной антенны были любезно предоставлены нам Г.А.Красинским. На основании имеющихся радиолокационных данных для каждой из планет (Меркурий, Венера, Марс) был создан банк радиолокационных наблюдений.

Геоцентрические координаты приемной и передающей антенны вычисляются по формулам [8]:

$$
x_{1}=\rho_{0} \cos \varphi^{\prime} \cos s, y_{1}=\rho_{0} \cos \varphi^{\prime} \sin s, z_{1}=\rho_{0} \sin s,
$$

где

$$
\begin{aligned}
& \rho_{0} \sin \varphi^{\prime}=\left(s+0.1568 \cdot 10^{-6} h\right) \sin \varphi, \rho_{0} \cos \varphi^{\prime}=\left(c+0.1568 \cdot 10^{-6} h\right) \cos \varphi, \\
& \operatorname{tg} \varphi^{\prime}=\left(0.993307+0.0011 \cdot 10^{-6} h\right) \operatorname{tg} \varphi, s_{m}=s_{0}+m+\mu(m+\lambda), \\
& s_{0}=6^{h} 40^{m} 18^{s} .130+8640184^{s} .635 T^{\prime}+0^{s} .0929 T^{\prime 2} .
\end{aligned}
$$

Здесь $T^{\prime}=d^{\prime} / 36525, \quad d^{\prime}=J D\left(T_{0}\right)-J D 2433282.5, T_{0}$ - всемирное время, $\lambda$ - долгота пункта наблюдения в часовой мере, $m$ - местное среднее время: $m=T_{0}+\lambda, \quad \mu=0.00274379093$.

Для удобства сопоставления данных наблюдений с теоретическими данными время излучения из всемирного переведено в эфемеридное и представлено в юлианских днях. В качестве единицы измерения временной задержки использовалась 1 мкс, при этом имеет место следующее соответствие: 6 мкс $\approx 1$ км.

Построение численной теории движения больших планет можно разбить на ряд самостоятельных этапов: а) определение начальных орбит или начальных данных координат и скоростей планет; б) численное интегрирование уравнений движения; в) сопоставление координат и скоростей планет, полученных из вычислений, с наблюдениями. Начальные данные координат планет были согласованы с данными координат, полученными на основе теории движения больших планет (Меркурий-Нептун), разработанной французским бюро долгот [9], где координаты и скорости вычислялись с помощью полиномов Чебышева. Выбор начального момента интегрирования был обусловлен тем, что вблизи этой даты имеются высокоточные радиолокационные наблюдения Меркурия, Венеры и Марса.

В таблице приведены начальные данные экваториальных координат и скоростей больших планет (Меркурий-Плутон) и Луны на начальный момент интегрирования, приведенные к эпохе 1950.0. Данным значениям координат и скоростей планет соответствуют следующие значения их обратных масс:

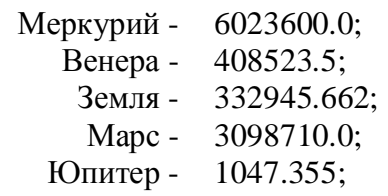

$$
\begin{aligned}
\text { Сатурн - } & 3498.5 \\
\text { Уран - } & 22869.0 \\
\text { Нептун - } & 19314.0 \\
\text { Плутон - } & 135200000.0 \\
\text { Луна - } & 27069007.13
\end{aligned}
$$

В дифференциальных уравнениях движения внутренних планет наряду с ньютоновскими членами учитывались релятивистские эффекты. Гелиоцентрические релятивистские уравнения в матричной форме имеют следующий вид [10]:

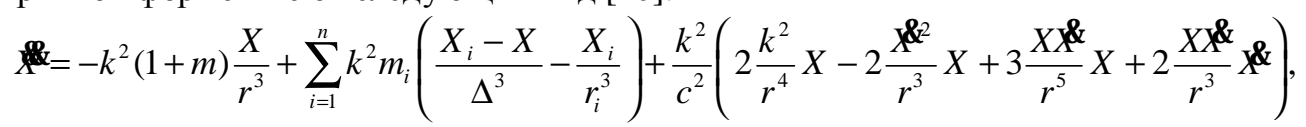

где $X$ - матрица-столбец с элементами $x, y, z ; X_{i}$ - матрица-столбец с элементами $x_{i}, y_{i}, z_{i}$; $m, x, y, z$ - масса и гелиоцентрические координаты возмущающей планеты; $m_{i}, x_{i}$ - массы и гелиоцентрические координаты возмущающих планет; $r, \Delta_{i}, r_{i}$ - расстояния, вычисляемые по формулам: $r^{2}=x^{2}+y^{2}+z^{2}, \Delta_{i}^{2}=\left(x_{i}-x\right)^{2}+\left(y_{i}-y\right)^{2}+\left(z_{i}-z\right)^{2}, r_{i}{ }^{2}=x_{i}{ }^{2}+y_{i}{ }^{2}+z_{i}{ }^{2} ; c-$ 


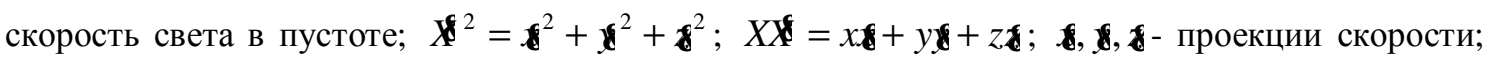
время, входящее в уравнение (3), координатное.

В уравнениях движения Луны следует учитывать возмущение от несферичности Земли. Потенциал для Земли вычисляется по следующей формуле [8]:

$$
u=\frac{k^{2} m}{r}\left\{1-\sum_{n=2}^{\alpha} J_{n}\left(\frac{r_{0}}{r}\right)^{n} P_{n}(\sin \varphi)+\sum_{n=2}^{\alpha} \sum_{k=1}^{n}\left(\frac{r_{0}}{r}\right)^{n} P_{n}^{(k)}(\sin \varphi)\left[c_{n k} \cos k \lambda+s_{n k} \sin k \lambda\right]\right\},
$$

где $m$ - масса Земли; $r_{0}$ - средний экваториальный радиус, $\varphi, \lambda$ - широта и долгота соответственно. $P_{n}$ и $P_{n}^{(k)}$ - полином и присоединенная функция Лежандра, причем

$$
P_{n}(z)=\frac{1}{2 n !} \frac{d^{n}\left(z^{2}-1\right)^{n}}{d z^{n}}, P_{n}^{(k)}(z)=\left(1-z^{2}\right)^{k / 2} \frac{d^{k} P_{n}(z)}{d z^{k}} .
$$

Коэффициенты $J_{n}, c_{n k}, s_{n k}$ зональных и тессеральных гармоник зависят от формы и распределения масс. Численные значения этих коэффициентов до шестнадцатого порядка включительно приведены в работе [11]. Система дифференциальных уравнений (3) с учетом (4), (5) являлась основной при изучении движения больших планет и Луны. Численное интегрирование уравнений движения проведено методом Эверхарта 23 порядка с шагом интегрирования 3 дня [12].

Основной целью при решении данной задачи являлось: получить точные координаты планет и Луны с помощью единого алгоритма, а также согласовать численную теорию движения планет как с радиолокационными наблюдениями, так и с данными BDL, которые, как известно, периодически корректировались на различные моменты времени.

Результаты представления разностей вычисленных и наблюденных запаздываний отраженного сигнала показаны на рис. 2-4, где ось абсцисс - время, а ось ординат - расстояние в километрах. Поверхности планет: Меркурия, Венеры и Марса - аппроксимированы сферами с радиусами 2439, 6050 и 3394 км соответственно. Величина астрономической единицы принималась равной 149597875 км, скорость света в вакууме $c=299792,456$ км/с. Ошибки в координатах планет в процессе эволюции могут складываться из погрешностей начальных смещений, неучтенных сил взаимодействия, погрешности метода.

Как видно из рис. 2-4, у Меркурия и Венеры отклонения вычисленных значений запаздывания отраженного сигнала от наблюденного на интервале времени 1964-1984 гг. находится в основном в пределах от -40 до +40 км, а для Марса - от -80 до +80 км. Если это расхождение с 1964 по 1970 гг. можно объяснить погрешностями, связанными с точностью радиолокационных наблюдений, то после 1970-го года точность наблюдений значительно возросла. По-видимому, полученные расхождения вычисленных и наблюденных запаздываний отраженного сигнала связаны либо с погрешностями начальных смещений в координатах и скоростях, либо с неточностью теории движения Луны, поскольку в процессе численного интегрирования от несферичности Земли учитывались лишь зональные гармоники. В заключение следует отметить, что полученные результаты являются предварительными и будут уточняться с устранением отмеченных недостатков.

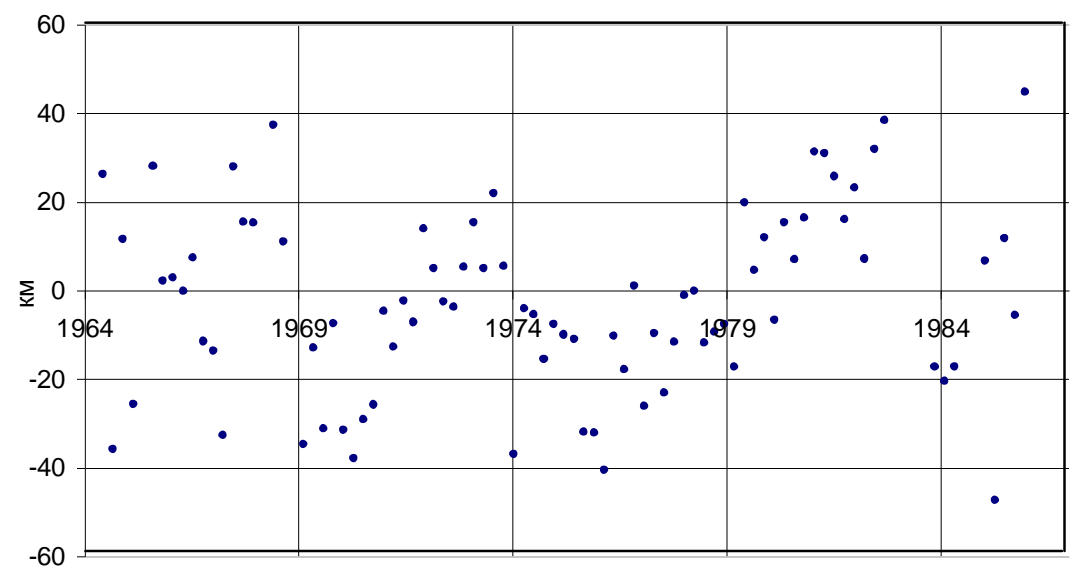

Р и с. 2. Меркурий, время запаздывания, 1964-1986 гг. 


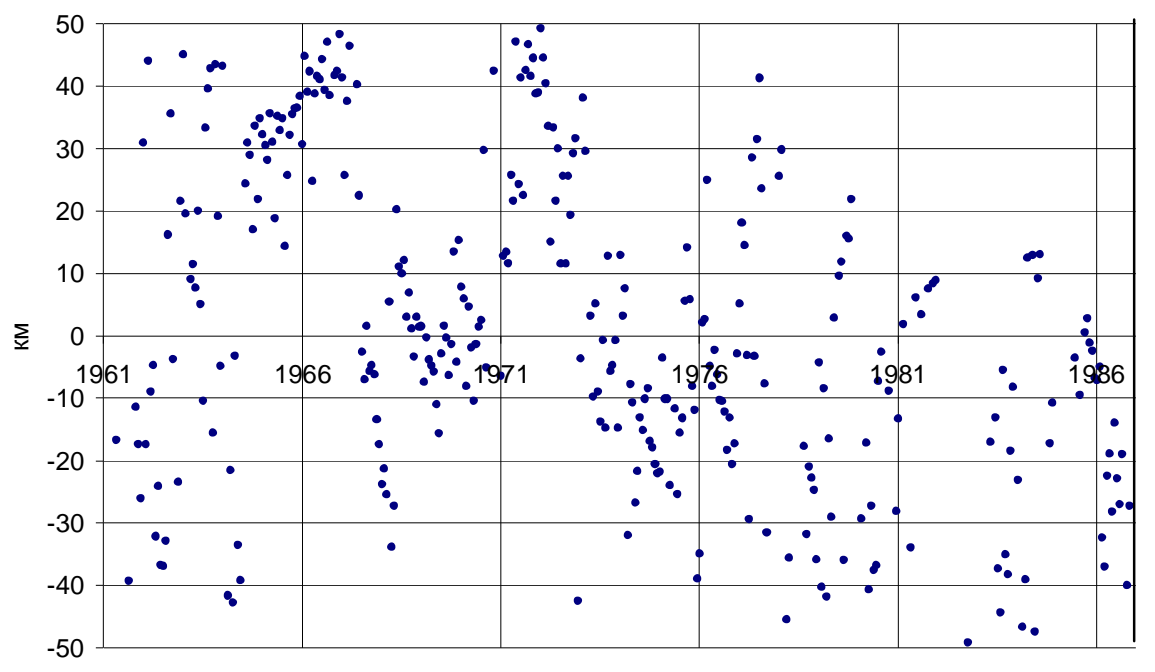

Р и с. 3. Венера, время запаздывания, 1964-1986 гг

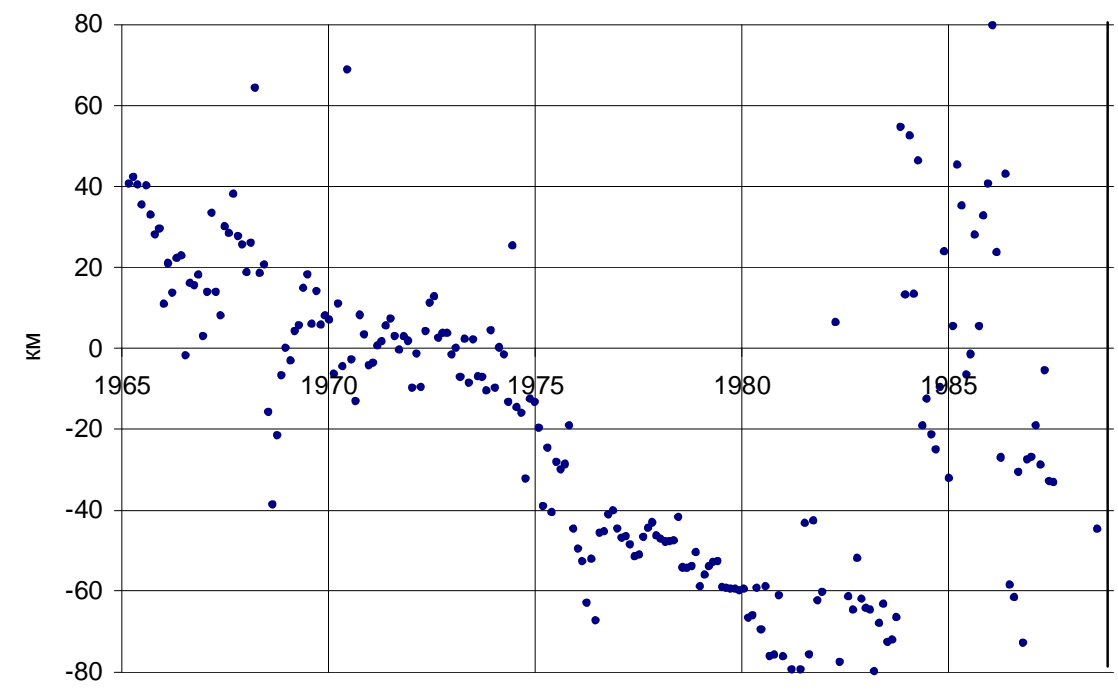

Р и с. 4. Марс, время запаздывания, 1964-1988 гг.

Прямоугольные координаты и скорости планет и Луны (JD=2438985.20524)

\begin{tabular}{|l|c|c|c|}
\hline \multirow{2}{*}{ Планеты } & \multicolumn{3}{|c|}{ Координаты, а.е. } \\
\cline { 2 - 4 } & $x$ & $y$ & $z$ \\
\hline Меркурий & 0.276782484324 & -0.266310923043 & -0.171054299101 \\
Венера & -0.556233837151 & -0.433477751614 & -0.160197180274 \\
Земля & 0.770853666014 & -0.603168291797 & -0.261560397747 \\
Луна & 0.773020767753 & -0.604552503210 & -0.262407129501 \\
Марс & -0.705414981930 & -1.228088178385 & -0.544766502473 \\
Юпитер & 1.315251047781 & 4.515557687907 & 1.905038796205 \\
Сатурн & 9.245711130382 & -2.499274047073 & -1.433026177260 \\
Уран & -17.635147608060 & 4.333491971666 & 2.147945785814 \\
Нептун & -19.889669767840 & -21.351321821290 & -8.245616118940 \\
Плутон & -26.231756160300 & 20.561529778100 & 14.443690294800
\end{tabular}


Окончание таблицы

\begin{tabular}{|l|c|c|c|}
\hline \multirow{2}{*}{ Планеты } & \multicolumn{3}{|c|}{ Скорости, а.е./сутки *100 } \\
\cline { 2 - 4 } & $\boldsymbol{\delta}$ & $\boldsymbol{\delta}$ & $\delta$ \\
\hline Меркурий & 1.548861897091 & 1.832079085761 & 0.821989341228 \\
Венера & 1.277015710772 & -1.398792934761 & -0.710976610121 \\
Земля & 1.087920376471 & 1.194908746617 & 0.518183941944 \\
Луна & 1.120669335550 & 1.237479538506 & 0.535092025352 \\
Марс & 1.293747728378 & -0.470640434995 & -0.250769890492 \\
Юпитер & -0.737746303257 & 0.206046599656 & 0.106426463933 \\
Сатурн & 0.133676565283 & 0.494197114637 & 0.198555281780 \\
Уран & -0.107650915515 & -0.364505590120 & -0.158192143258 \\
Нептун & 0.234294794744 & -0.186442474920 & -0.082268481545 \\
Плутон & 0.131572184319 & -0.261983661854 & -0.042705125436 \\
\hline
\end{tabular}

\section{БИБЛИОГРАФИЧЕСКИЙ СПИСОК}

1. Standish E.M. The observational basis for JPL'S DE200 the planetary ephemeredes of the Astronomical Almanac // Astronom Astrophys. 1990. 233. P. 252-271.

2. Standish E.M., Newhall X.X., Williams J.G. and Folkner W.E. JPL Planetary and Lunar Ephemerides, DE403/LE403 // JPL IOM. 314. 1995. P. 10-127.

3. Standish E.M. JPL Planetary and Lunar Ephemerides, DE403/LE403 // JPL IOM. 312. 1998. P. 1-18.

4. Красинский Г.А., Питьева Е.В., Свешников М.Л., Свешникова Е.С. Уточнение эфемерид внутренних планет и Луны по радиолокационным, лазерным и мередианным измерениям 1961-1980 гг. // Бюлл. ИТА. XV. №3(166). 1989. C. 145-164.

5. Александров Ю.Н., Кузнещов Б.Н., Петров Г.М., Ржига О.Н. Методика радиолокационных астрономических наблюдений. // Астрон. Журнал, Т.49, 1972, с. 175-185.

6. Кислик М.Д., Комока Ю.В., акад. Котельников В.А., Петров В.Ф. Определение орбит Земли и Венеры, астрономической единицы и радиуса Венеры на основе радиолокационных наблюдений Венеры в 1962-1977 гг. // ДАН. 241. №5. 1978. С. 1046-1049.

7. Котельников B.A. и др. Результаты измерений времени запаздывания и доплеровской поправки, полученные в радиолокационных наблюдениях Венеры в 1962, 1964, 1969, 1970 и 1972 гг. // Астрон. Журнал. Т. 50. Вып. 5. 1973. С.836-848.

8. Абалакин В.К., Аксенов Е.П., Гребенников Е.А., Рябов Ю.А. Справочное руководство по небесной механике и астродинамике. М.: Наука. 1979. 584 с.

9. Dretagnon P. Teorie du movement de l'ensemble des planets solution VSOP82 // Astronom Astrophys. 1990. 114. P. 278-288.

10. Брумберг В.А. Релятивистская небесная механика. М.: Наука. 1972. 383 с.

11. Грушинский Н.П. Основы гравиметрии. М.: Наука. 1983. 351 с.

12. Everhart E. Implist single methods for integrating orbits // Central Mechanics. 1974. 10. P. 35-55.

Поступила 25.11.2003 2. 